\title{
Análisis de la veracidad de determinadas creencias asociadas habitualmente al entrenamiento de fuerza. Una revisión narrativa \\ Analysis of the veracity of certain beliefs frequently associated to resistance training. A narrative review
}

Pablo Prieto Gonzalez, Peter Sagat, Mehdi Ben Brahim, Jaromir Sedlacek Prince Sultan University (Arabia Saudí)

\begin{abstract}
Resumen. El objetivo del estudio fue contrastar la veracidad de las siguientes creencias:1-El entrenamiento de fuerza y resistencia es incompatible. 2-El entrenamiento de fuerza limita la flexibilidad. 3-Las rutinas divididas son más eficaces que las de cuerpo entero. 4-El entrenamiento de fuerza no es útil para la pérdida de peso, o la mejora de la composición corporal. Se realizó una búsqueda en las siguientes bases de datos: ProQuest, Google Scholar, Scopus, ScienceDirect y Web of Science. Los criterios de selección fueron: a) Artículos escritos en Español o en Inglés. b) Investigaciones primarias con metodología experimental o cuasi-experimental. c) Escritos entre el año 2015 y 2019, salvo para los apartados 2 y 3, que se amplió hasta 2000 y 1990 respectivamente, debido a la escasez de publicaciones. d) La población objeto de estudio fueron adultos sanos que no practicaban deporte a nivel profesional o semiprofesional. e) Artículos que recogen exclusivamente adaptaciones logradas mediante una intervención con entrenamiento. Analizados los estudios, se pudo determinar que en adultos sanos: 1- El entrenamiento de fuerza y resistencia es compatible. 2- El entrenamiento de fuerza no deteriora la flexibilidad, y podría incluso mejorarla. 3- En virtud de los estudios existente, las rutinas divididas y las de cuerpo entero son igualmente eficaces para incrementar la fuerza. Las rutinas de cuerpo entero podrían generar mayor hipertrofia muscular. 4- El entrenamiento de fuerza es eficaz en la mejora de la composición corporal, y podría tener un impacto positivo en biomarcadores cardiovasculares y metabólicos.
\end{abstract}

Palabras clave: Entrenamiento concurrente, resistencia, flexibilidad, fuerza.

\begin{abstract}
The purpose of the study was to verify the veracity of the following beliefs: 1-Resistance and endurance training are incompatible. 2-Resistance training reduces flexibility. 3-Split body routines are more effective than full-body routines. 4-Resistance training is not useful neither in weight loss programs, nor to change body composition. The following databases were searched: ProQuest, Google Scholar, Scopus, ScienceDirect and Web of Science. The selection criteria were: a) Articles written in Spanish or in English b) Primary research following an experimental or quasi-experimental methodology c) Written between 2015 and 2019, except for section 2 and 3, which was extended until 2000 and 1990 respectively, due to the shortage of publications d) The target population of study were healthy adults who did not practice sports at the professional or semi-professional level e) Papers which include only adaptations achieved through training interventions. Once the studies were analyzed, it was concluded that in healthy adults: 1-The combination of resistance and endurance training is compatible. 2- Strength training does not decrease flexibility, and it could even improve it. 3- On the basis of existing studies, split and full-body routines are equally effective in improving strength. Full-body routines could generate higher muscle hypertrophy. 4- Strength training is effective in improving body composition, and could make a positive impact on cardiovascular and metabolic biomarkers.
\end{abstract}

Key words: Concurrent training, endurance, flexibility, strength.

\section{Introducción}

En las últimas décadas, el entrenamiento de fuerza ha alcanzado un elevado grado de popularidad (Weier, Pearce, \& Kidgell, 2012). El desarrollo de esta capacidad es cada vez más común entre la población no sedentaria. De este modo, y para satisfacer a un grupo cada vez más numeroso y heterogéneo de personas, las actividades, medios y métodos de entrenamiento destinados a mejorar los niveles de fuerza se han diversificado. En la actualidad, si bien es cierto que la mayor parte de la población que practica actividad física lo hace para mejorar su salud y su condición física (Campos, González-Víllora, González-Gómez, \& Martins, 2018), los trabajos de fuerza se realizan además con otros objetivos, entre los que cabe destacar: La mejora del rendimiento deportivo, de la autoimagen, el culto al cuerpo, la prevención de lesiones y la rehabilitación (Pronk, Bender, \& Katz, 2016).

El creciente interés por el entrenamiento de fuerza dentro de la población que practica actividad física se ha visto también acompañado de la aparición de numerosos estudios

Fecha recepción: 13-01-19. Fecha de aceptación: 17-10-19

Pablo Prieto Gonzalez

pabloccjb@gmail.com científicos realizados por profesionales vinculados a las ciencias de la actividad física y del deporte. Aun así, existen centros deportivos en los que los entrenamientos de fuerza no se ejecutan siempre de acuerdo con la evidencia científica, sino que responden en ocasiones a modas pasajeras, de modo que se manejan conceptos erróneos (Fry \& Newton, 2006; Thompson, 2018). Esto favorece la aparición de diversos mitos y creencias. De acuerdo con López-Miñarro (2002), dichos mitos y creencias surgen por los siguientes motivos:

-Desconocimiento por parte de buena base de la población de las bases biológicas del ejercicio físico.

-Publicidad: Mensajes distorsionados emitidos por los fabricantes de «productos milagrosos», que nos indican las bondades de su producto la hora de incrementar la masa muscular, modificar la composición corporal, perder peso o incrementar el rendimiento deportivo.

-Medios de comunicación: Se difunde información relacionada con la actividad física y el deporte que carece de rigor científico.

-Intrusismo profesional y existencia de trabajadores con una formación inadecuada, que

prestan sus servicios profesionales en empresas o centros deportivos.

Las creencias vinculadas a la práctica diaria de trabajos 
de fuerza, lejos de desaparecer, se mantienen en la actualidad con gran firmeza (Kraemer, \& Fleck, 2005). Varios autores han constatado la existencia dichas creencias, siendo las más recurrentes las que se indican a continuación:

a) Las personas que realizan trabajos de fuerza, a menudo no desarrollan otras capacidades físicas básicas como la resistencia (García-Orea, Heredia, Aguilera, Da Silva, \& Del Rosso, 2016) o la flexibilidad (Del Río, Flores, González, Barajas, Valencia, \& Gómez, 2015), en virtud de una presunta incompatibilidad entre dichas capacidades.

b) En los programas de adelgazamiento, el entrenamiento aeróbico es el gran protagonista (Torres Luque, GarcíaMartos, Villaverde, \& Garatachea, 2010), de modo que se excluye habitualmente el entrenamiento fuerza.

c) A la hora de diseñar la estructura de la sesión de entrenamiento de fuerza, las rutinas dividas son las más eficaces para conseguir adaptaciones vinculadas a la hipertrofia muscular (Peterson, Rhea, \& Alvar, 2004; Schoenfeltd, Ogborn, \& Krieger, 2016). Por tanto, resulta necesario contrastar estas aseveraciones con la literatura científica. En éste sentido, en la actualidad, no existen artículos de revisión en los que se haya sintetizado la influencia que ejerce el entrenamiento de fuerza sobre la flexibilidad, ni que tipo de rutinas son más eficaces, las de cuerpo entero o las rutinas divididas. En cambio, si han sido publicadas dos revisiones sistemáticas recientes en las que se ha tratado de verificar la compatibilidad del entrenamiento conjunto de fuerza y resistencia (Gäbler, Prieske, Hortobágyi \& Granacher, 2018; Sabag, Najafi, Michael, Esgin, Halaki \& Hackett, 2018). Pero la primera de ellas se centra exclusivamente en menores de 18 años, y la segunda tan solo analiza la compatibilidad entre el entrenamiento interválico de alta intensidad y el entrenamiento de fuerza. También existen dos revisiones sistemáticas publicadas en los últimos cinco años en las que se ha estudiado la importancia del entrenamiento de fuerza en los programas de pérdida de peso mediante revisiones sistemáticas (Balsalobre-Fernández \& Tejero-González, 2015; Ten Hoor, Kok, Peters, Frissen, Schols, \& Plasqui, 2017). Sin embargo, en el primero de ellos, el grupo de población analizado fue exclusivamente personas con obesidad, y en el segundo personas con sobrepeso u obesidad.

En este contexto, se procedió a realizar una revisión literaria para constatar la veracidad de las siguientes creencias:

1. El entrenamiento conjunto de fuerza y resistencia es incompatible, de modo que entrenar ambas capacidades de forma simultánea dificulta la consecución de las adaptaciones propias de los trabajos exclusivos de fuerza y resistencia.

2. El entrenamiento de fuerza deteriora los niveles de flexibilidad.

3. Cuando el objetivo del entrenamiento es la hipertrofia muscular, las rutinas dividas son más eficaces que las rutinas de cuerpo entero.

4. El entrenamiento de fuerza carece de utilidad en los programas de pérdida de peso o de cambio de composición corporal.

El estudio se centró exclusivamente en adultos sanos, que no practicaban deporte a nivel profesional o semiprofesional.

\section{Método}

Para la selección de artículos se emplearon las siguientes bases de datos: ProQuest, Google Scholar, Scopus, ScienceDirect y Web of Science. Los términos de búsqueda fueron agrupados en cuatro apartados, en función de los cuatro aspectos que se analizaron en el presente estudio, tal y como se detalla en la tabla 1.

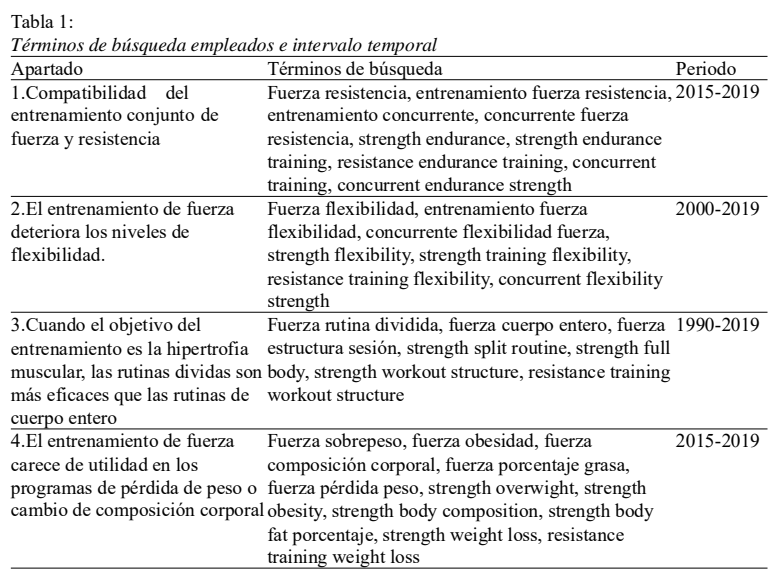

Los criterios de inclusión utilizados en la selección de artículos fueron los siguientes:

a) Artículos escritos en Español y en Inglés. b) Artículos que recogen los resultados de investigaciones primarias, y que siguen una metodología experimental o cuasi experimental. c) Artículos escritos entre los años 2015 y 2019, excepto en el caso del apartado número 2 (el entrenamiento de fuerza deteriora los niveles de flexibilidad) que se amplió hasta el año 2000, y en el apartado 3 (rutina dividida versus rutina de cuerpo entero), que se amplió hasta el año 1990 debido a la escasez de publicaciones vinculadas a estos dos contenidos. d) Investigaciones realizadas con personas adultas (con edades comprendidas entre los 18 a 64 años), que no practicaban deporte a nivel profesional o semiprofesional, y que no padecían ninguna enfermedad crónica ni presentaban obesidad (IMC <30). e) Artículos que recogen exclusivamente adaptaciones logradas por medio de una intervención con entrenamiento. Se excluyeron los artículos en los que se analizaron los efectos agudos de una sesión de entrenamiento de fuerza, y aquellos en los que se estudiaron exclusivamente las adaptaciones a nivel fisiológico. También se descartaron los artículos en los que

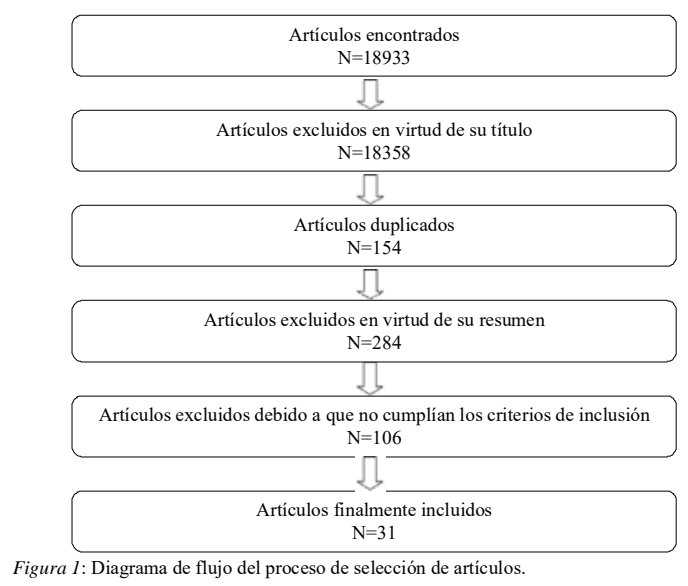


se realizaron intervenciones dietético-nutricionales, dado que podrían modificar el resultado de la investigación.

De los 18933 encontrados en las cinco bases de datos, 18902 fueron excluidos en función de su título, de su resumen, o bien porque no cumplían los criterios de inclusión o estaban duplicados (ver figura 1).

\section{Análisis y discusión}

El entrenamiento concurrente de fuerza y resistencia es incompatible, de modo que entrenar ambas capacidades de forma simultánea dificulta la consecución de las adaptaciones propias del trabajo de fuerza y de resistencia

Compatibilizar el entrenamiento de fuerza con el de resistencia ha generado siempre una gran controversia. A menudo se ha especulado con la posibilidad de que el entrenamiento concurrente de fuerza y resistencia no genera las mismas adaptaciones que el entrenamiento por separado de ambas capacidades. Los argumentos que se han esgrimido a la hora de señalar esta posible incompatibilidad son los siguientes: (Leveritt, Abernethy, Barry \& Logan, 2003):

-En el caso de entrenar fuerza y resistencia en el mismo día, la fatiga aguda de la sesión inicial comprometería la carga de trabajo de la sesión de entrenamiento posterior, y por consiguiente, las adaptaciones que se pudiesen alcanzar.

-Se podría producir una inhibición en la consecución de adaptaciones debido al sobrentrenamiento.

-Las adaptaciones neuromusculares que se logran a través del entrenamiento de fuerza difieren de las logradas con el entrenamiento de resistencia.

En este sentido, Badillo y Rivas (2002), señalan que las adaptaciones que se logran a través del entrenamiento de fuerza y resistencia son opuestas. Indican que el entrenamiento de fuerza: a) Reduce la densidad mitocondrial. b) Estabiliza o reduce la red capilar. c) Disminuye la actividad de las enzimas oxidativas. d) Incrementa el tamaño de la fibra muscular. e) Aumenta la capacidad contráctil y mejora la fuerza y la potencia anaeróbica. Por contra, el entrenamiento de resistencia: a) Incrementa la densidad mitocondrial y capilar, aumentando la actividad de las enzimas oxidativas. b)

Estabiliza o incluso disminuye el tamaño de las fibras musculares, disminuyendo así su capacidad contráctil y la velocidad de acortamiento de las fibras tipo II. c) Reduce la aplicación de fuerza por unidad de tiempo de todas las fibras.

Por lo tanto, a priori, sería compresible que tanto entrenadores como preparadores físicos, deportistas y profesionales de la actividad física y del deporte tengan reservas a la hora de realizar trabajos de fuerza y resistencia dentro la misma sesión. Y este es un tema de gran relevancia, pues son muchas las modalidades deportivas en las que ambas capacidades son necesarias. Pero afortunadamente, existen numerosas publicaciones en la literatura científica en las que se ha analizado esta cuestión en profundidad, y permiten extraer conclusiones claras. En la tabla 2 se exponen los resultados de 15 estudios en los que se aplicó un entrenamiento concurrente de fuerza y resistencia.

En 14 de las 15 investigaciones el entrenamiento concurrente generó las adaptaciones propias del trabajo exclusivo de fuerza y de resistencia. Tan solo en el estudio realizado por Schumann et al. (2016) se constató que la adicción de un programa de un entrenamiento de fuerza al programa de entrenamiento de resistencia de corredores aficionados, no generó hipertrofia ni incrementó los niveles de fuerza máxima. Los autores de esta investigación entienden que realizar una sesión de fuerza después del entrenamiento de resistencia imposibilitó la consecución de las adaptaciones propias de los trabajos de fuerza, debido a la fatiga acumulada. Pero al margen de esta investigación, los hallazgos alcanzados en el resto de estudios analizados ofrecen información relevante, que permite diseñar estrategias adecuadas para evitar que se produzcan interferencias entre las dos capacidades. En este sentido, Küüsmaa-Schildt et al. (2017) comprobaron que la hora del día en la que se realiza el entrenamiento concurrente no influye en las adaptaciones logradas. En cambio, en otro estudio previo (Küüsmaa-Schildt et al. (2016) se constató que los entrenamientos concurrentes realizados por la tarde generan mayores niveles de hipertrofia. Por su parte, en los estudios de Tomiya et al. (2017), Eklund et al. (2016), Robineau et al. (2016) y Eklund et al. (2015) se pudo comprobar que los entrenamientos concurrentes son más efectivos cuando las sesiones de fuerza y de resistencia se realizan en días diferentes. En caso de llevarse a cabo el mismo día, realizar la sesión de fuerza antes que la de resistencia es más efectivo para lograr adaptaciones de fuerza. Por contra, el orden inverso favorece las adaptaciones de resistencia (Eklund et al., 2015; Küüsmaa-Schildt et al., 2017; Robineau et al., 2016).

Con respecto a la carga de trabajo, Fyfe et al. (2016), mediante una investigación en la que se aplicaron dos entrenamientos concurrentes diferentes, llegaron a la conclusión de que un elevado volumen de trabajo de resistencia, es más susceptible de atenuar las adaptaciones de fuerza que una elevada intensidad. En esta misma línea, Varela-Sanz et al. (2017) pudieron verificar que un entrenamiento polarizado (que combina sesiones de alto volumen y baja intensidad, con sesiones de bajo volumen y alta intensidad), genera menos interferencias que un entrenamiento tradicional. Finalmente, Rosell et al. (2017) constataron que cuando en el entrenamiento concurrente se realizan trabajos de fuerza de intensidad elevada (70-85\% 1RM), además de mejorar la fuerza máxima y la capacidad aeróbica, se mejora la fuerza explosiva.

El entrenamiento de fuerza supone una merma en los niveles de flexibilidad

Una de las creencias más extendidas en el ámbito de la actividad física y del deporte es asumir que el entrenamiento de fuerza reduce los niveles de flexibilidad. Para aclarar esta cuestión, se han analizado los resultados de cuatro estudios en los que se examinó la interacción entre ambas capacidades. En la tabla número 3 se muestran los resultados de dichos estudios.

Aunque la actual evidencia científica es un tanto escasa, y es preciso realizar más estudios en el futuro, en ninguna de las investigaciones analizadas el entrenamiento de fuerza supuso una reducción de los niveles de flexibilidad. Es más, en tres de estos cuatro estudios, se pudo comprobar que el entrenamiento de fuerza mejoró la flexibilidad (Azeem \& Hashem, 2019; Morton at al. 2011; Santos et al., 2010). Por 
Tabla 2

\begin{tabular}{|c|c|c|c|c|c|c|}
\hline Estudio & $\mathrm{N}$ & Grupo de Población & Objetivo & $\begin{array}{l}\text { Diseño } \\
\text { Experimental }\end{array}$ & Duración & Resultados \\
\hline $\begin{array}{l}\text { Amaro-Gahete, } \\
\text { De-la-O, Jurado- } \\
\text { Fasoli, Dote- } \\
\text { Montero, } \\
\text { Gutiérrez, Ruiz y } \\
\text { Castillo, } 2019\end{array}$ & 89 & $\begin{array}{l}\text { Adultos sedentarios. } \\
\text { Edad: } 53.7 \pm 5.1\end{array}$ & $\begin{array}{l}\text { Comprobar el efecto } \\
\text { de diferentes } \\
\text { modalidades de } \\
\text { ejercicio }\end{array}$ & $\begin{array}{l}\text {-Grupo entrenamiento concurrente basado } \\
\text { en las recomendaciones de la OMS (PAR) } \\
\text {-Grupo de entrenamiento de intervalos de } \\
\text { alta intensidad (HITT) } \\
\text {-Grupo de entrenamiento de intervalos de } \\
\text { alta intensidad combinado con } \\
\text { electroestimulación de cuerpo completo } \\
\text { (WB-EMS) }\end{array}$ & $\begin{array}{l}12 \text { semanas. PAR: Tres sesiones } \\
\text { semanales de entrenamiento. } \\
\text { HITT y WB-EMS: Dos sesiones } \\
\text { semanales de entrenamiento } \\
\end{array}$ & $\begin{array}{l}\text { Los tres grupos mejoraron } \\
\text { significativamente su condición } \\
\text { fisica general. WB-EMS obtuvo } \\
\text { resultados ligeramente } \\
\text { superiores en algunas variables, } \\
\text { pero no fueron significativos }\end{array}$ \\
\hline $\begin{array}{l}\text { Karatrantou, } \\
\text { Gerodimos, } \\
\text { Häkkinen y } \\
\text { Zafeiridis, } 2017\end{array}$ & 54 & Mujeres. Edad: $46.7 \pm 4.5$ & $\begin{array}{l}\text { Comprobar el efectos } \\
\text { de dos entrenamientos } \\
\text { concurrentes (uno } \\
\text { seriado y otro } \\
\text { integrado) sobre la } \\
\text { condición fisica y la } \\
\text { salud }\end{array}$ & $\begin{array}{l}\text {-Grupo entrenamiento seriado. Realizó el } \\
\text { trabajo de fuerza antes que el de } \\
\text { resistencia (SCG) } \\
\text {-Grupo entrenamiento integrado. El } \\
\text { trabajo de fuerza y resistencia se realizó } \\
\text { en un orden preestablecido (ICG) } \\
\text {-Grupo control CG }\end{array}$ & Tres meses & $\begin{array}{l}\text { Tanto SCG como ICG mejoraron } \\
\text { su condición física, composición } \\
\text { corporal, función respiratoria, } \\
\text { tensión arterial y equilibrio sin } \\
\text { diferencias significativas entre } \\
\text { ambos grupos experimentales }\end{array}$ \\
\hline $\begin{array}{l}\text { Tomiya, Kikuchi } \\
\text { y Nakazato, } 2017\end{array}$ & 14 & $\begin{array}{l}\text { Mujeres y hombres } \\
\text { jóvenes. Edad: Grupo CT: } \\
21.8 \pm 0.7 \text {. Grupo SEP: } \\
22.1 \pm 0.7\end{array}$ & $\begin{array}{l}\text { Examinar el efecto de } \\
\text { un entrenamiento } \\
\text { concurrente } \\
\text { compuesto por un } \\
\text { entrenamiento de } \\
\text { fuerza de tren } \\
\text { superior más trabajo } \\
\text { aeróbico en bicicleta } \\
\end{array}$ & $\begin{array}{l}\text {-Grupo entrenamiento concurrente (fuerza } \\
\text { en tren superior + bici) en el mismo día } \\
\text { (CT) } \\
\text {-Grupo entrenamiento concurrente (fuerza } \\
\text { en tren superior y bici) en días diferentes } \\
\text { (SEP) }\end{array}$ & $\begin{array}{l}\text { Ocho semanas. Dos sesiones } \\
\text { semanales }\end{array}$ & $\begin{array}{l}\text { Ambos grupos incrementaron su } \\
\text { fuerza máxima, consumo } \\
\text { máximo de oxígeno y masa } \\
\text { muscular. Sin embargo, el grado } \\
\text { de hipertrofia fue superior en } \\
\text { SEP }\end{array}$ \\
\hline $\begin{array}{l}\text { Karatrantou, } \\
\text { Gerodimos, } \\
\text { Häkkinen y } \\
\text { Zafeiridis, } 2017\end{array}$ & 54 & $\begin{array}{l}\text { Mujeres con buen estado } \\
\text { de salud, que no habían } \\
\text { practicado actividad física } \\
\text { el último año. Edad: } \\
46.7 \pm 4.5\end{array}$ & $\begin{array}{l}\text { Comparar el efecto de } \\
\text { dos programas de } \\
\text { entrenamiento } \\
\text { concurrente }\end{array}$ & $\begin{array}{l}\text {-Grupo entrenamiento concurrente seriado } \\
\text { (SCG): Fuerza siempre antes que } \\
\text { resistencia } \\
\text {-Grupo entrenamiento integrado (ICG): } \\
\text { Las sesiones de fuerza y resistencia se } \\
\text { alternaron en un orden preestablecido }\end{array}$ & $\begin{array}{l}\text { Tres meses. Tres sesiones } \\
\text { semanales }\end{array}$ & $\begin{array}{l}\text { Ambos grupos obtuvieron } \\
\text { incrementos similares en sus } \\
\text { niveles de fuerza, resistencia, } \\
\text { flexibilidad, equilibrio, tejido } \\
\text { magro y función respiratoria }\end{array}$ \\
\hline $\begin{array}{l}\text { Küüsmaa-Schildt, } \\
\text { Eklund, Avela, } \\
\text { Tuomas } \\
\text { Rytkönen, } \\
\text { Newton, } \\
\text { Izquierdo y } \\
\text { Häkkinen, } 2017\end{array}$ & 51 & $\begin{array}{l}\text { Mujeres físicamente } \\
\text { activas con buen estado } \\
\text { de salud. Edad: } 32.3 \pm 5.6\end{array}$ & $\begin{array}{l}\text { Comparar el efecto de } \\
\text { cuatro programas de } \\
\text { entrenamiento } \\
\text { concurrente diferentes }\end{array}$ & $\begin{array}{l}\text {-Grupo fuerza + resistencia por la mañana } \\
\text { (SEm) } \\
\text {-Grupo resistencia + fuerza por la mañana } \\
(\text { ESm) } \\
\text {-Grupo fuerza + resistencia por la tarde } \\
\text { (SEe) } \\
\text {-Grupo resistencia + fuerza por la tarde } \\
\text { (ESe) } \\
\text {-Grupo control }\end{array}$ & $\begin{array}{l}24 \text { semanas. Dos sesiones de } \\
\text { entrenamiento semanales las } 12 \\
\text { primeras semanas, y en las doce } \\
\text { últimas, cinco sesiones de } \\
\text { entrenamiento cada dos semanas }\end{array}$ & $\begin{array}{l}\text { Las adaptaciones logradas no se } \\
\text { vieron afectadas por la hora de } \\
\text { entrenamiento, pero si por el } \\
\text { orden en el que se realizaron las } \\
\text { sesiones de fuerza y resistencia. } \\
\text { SEe obtuvo las mayores mejoras } \\
\text { en fuerza y ESm y ESe las } \\
\text { mayores mejoras en resistencia }\end{array}$ \\
\hline $\begin{array}{l}\text { Eklund, Häkkinen } \\
\text { A, Laukkanen, } \\
\text { Balandzic, } \\
\text { Nyman } \\
\text { y Häkkinen K, } \\
2016\end{array}$ & $\begin{array}{l}140, \text { de los } \\
\text { que } 39 \\
\text { fueron } \\
\text { excluidos }\end{array}$ & $\begin{array}{l}\text { Personas activas y con } \\
\text { buen estado de salud. } \\
\text { Edad: } 18-40\end{array}$ & $\begin{array}{l}\text { Analizar el efecto de } \\
\text { tres entrenamientos } \\
\text { concurrentes sobre la } \\
\text { condición física, la } \\
\text { composición corporal } \\
\text { y el perfil lipídico }\end{array}$ & $\begin{array}{l}\text {-Entrenamiento de fuerza y resistencia en } \\
\text { diferentes días (DD) } \\
\text {-Entrenamiento de ambas capacidades el } \\
\text { mismo día, precediendo la resistencia a la } \\
\text { fuerza (ES) } \\
\text {-Entrenamiento de ambas capacidades el } \\
\text { mismo día, precediendo la fuerza a la } \\
\text { resistencia (SE) }\end{array}$ & $\begin{array}{l}24 \text { semanas. Primeras } 12 \\
\text { semanas: Cada semana DD } \\
\text { realizó dos sesiones de } \\
\text { entrenamiento concurrente y ES } \\
\text { y SE dos sesiones de fuerza y } \\
\text { dos de resistencia. Últimas } 12 \\
\text { semanas: Cada dos semanas DD } \\
\text { efectuó cinco sesiones de } \\
\text { entrenamiento concurrente, y ES } \\
\text { y SE cinco sesiones de fuerza y } \\
\text { cinco de resistencia }\end{array}$ & $\begin{array}{l}\text { Los tres protocolos son válidos } \\
\text { para mejorar la condición fisica } \\
\text { y el porcentaje de tejido magro. } \\
\text { El protocolo DD condujo a una } \\
\text { disminución de la masa grasa y } \\
\text { podría ser útil además para } \\
\text { prevenir enfermedades } \\
\text { cardiovasculares y metabólicas }\end{array}$ \\
\hline $\begin{array}{l}\text { Fyfe, Bartlett, } \\
\text { Hanson, Stepto y } \\
\text { Bishop, } 2016\end{array}$ & 23 & $\begin{array}{l}\text { Hombres físicamente } \\
\text { activos. Edad: } 29.6 \pm 5.5\end{array}$ & $\begin{array}{l}\text { Comparar el efecto de } \\
\text { dos entrenamientos } \\
\text { concurrentes distintos } \\
\text { con un entrenamiento } \\
\text { de fuerza }\end{array}$ & $\begin{array}{l}\text {-Entrenamiento interválico de alta } \\
\text { intensidad + entrenamiento de fuerza } \\
\text { (HIT+RT) } \\
\text {-Entrenamiento continuo de intensidad } \\
\text { moderada + entrenamiento de fuerza } \\
\text { (MICT+RT) } \\
\text {-Entrenamiento de fuerza (RT) }\end{array}$ & $\begin{array}{l}\text { Ocho semanas. Tres sesiones } \\
\text { semanales }\end{array}$ & $\begin{array}{l}\text { HIT y MICT no atenuaron la } \\
\text { fuerza máxima en el tren } \\
\text { superior, pero si en el tren } \\
\text { inferior. Ambos protocolos } \\
\text { atenuaron además las mejoras en } \\
\text { el salto con contramovimiento. } \\
\text { El volumen de trabajo podría } \\
\text { atenuar en mayor medida que la } \\
\text { intensidad las ganancias de } \\
\text { fuerza }\end{array}$ \\
\hline $\begin{array}{l}\text { Küüsmaa-Schildt } \\
\text { et al., } 2016\end{array}$ & $\begin{array}{l}72 \text { iniciaron } \\
\text { el estudio, y } \\
\text { solo } 42 \text { lo } \\
\text { finalizaron }\end{array}$ & $\begin{array}{l}\text { Hombros sanos que no } \\
\text { practicaron } \\
\text { entrenamientos ni de } \\
\text { fuerza ni de resistencia el } \\
\text { año previo al estudio }\end{array}$ & $\begin{array}{l}\text { Analizar el efecto de } \\
\text { cuatro entrenamientos } \\
\text { concurrentes sobre el } \\
\text { rendimiento físico, la } \\
\text { hipertrofia muscular y } \\
\text { las concentraciones } \\
\text { séricas de testosterona } \\
\text { y cortisol diurnas en } \\
\text { reposo }\end{array}$ & $\begin{array}{l}\text {-Entrenamiento de fuerza }+ \text { resistencia por } \\
\text { la mañana }(\mathrm{mS}+\mathrm{E}) \\
\text {-Entrenamiento de resistencia }+ \text { fuerza por } \\
\text { la mañana }(\mathrm{mE}+\mathrm{S}) \\
\text {-Entrenamiento de fuerza }+ \text { resistencia por } \\
\text { la tarde }(\mathrm{eS}+\mathrm{E}) \\
\text {-Entrenamiento de resistencia + fuerza por } \\
\text { la tarde }(\mathrm{eE}+\mathrm{S}) \text {. }\end{array}$ & $\begin{array}{l}24 \text { semanas. En las } 12 \text { primeras, } \\
\text { se realizaron dos sesiones } \\
\text { semanales, y en las } 12 \text { últimas, } \\
\text { se realizaron cinco sesiones cada } \\
\text { dos semanas }\end{array}$ & $\begin{array}{l}\text { El entrenamiento concurrente } \\
\text { por la tarde podría ser más eficaz } \\
\text { para } \\
\text { incrementar la masa muscular. } \\
\text { Entrenar la resistencia antes que } \\
\text { la fuerza podría ser más } \\
\text { beneficioso para el rendimiento } \\
\text { de resistencia. El orden y la hora } \\
\text { de entrenamiento solo influye en } \\
\text { los resultados a partir de las } 12 \\
\text { semanas }\end{array}$ \\
\hline
\end{tabular}




\begin{tabular}{|c|c|c|c|c|c|c|}
\hline $\begin{array}{l}\text { Laird, Elmer, } \\
\text { Barberio, Salom, } \\
\text { Lee y Pascoe, } \\
2016\end{array}$ & 28 & $\begin{array}{l}\text { Mujeres que practicaban } \\
\text { actividad fisica. Edad: } \\
20.3 \pm 1.7\end{array}$ & $\begin{array}{l}\text { Comparar el efecto de } \\
\text { un entrenamiento } \\
\text { concurrente (interval } \\
\text { corto + fuerza) con un } \\
\text { entrenamiento de } \\
\text { fuerza }\end{array}$ & $\begin{array}{l}\text {-Grupo entrenamiento interválico corto + } \\
\text { fuerza (CST) } \\
\text {-Grupo entrenamiento de fuerza (RT) } \\
\end{array}$ & $\begin{array}{l}11 \text { semanas. Tres sesiones } \\
\text { semanales }\end{array}$ & $\begin{array}{l}\text { Ambos grupos mejoraron su } \\
\text { fuerza máxima y su potencia } \\
\text { aeróbica. Solo CST mejoró la } \\
\text { capacidad aeróbica. Por tanto, se } \\
\text { verificó que el entrenamiento } \\
\text { interválico no generó } \\
\text { interferencias }\end{array}$ \\
\hline $\begin{array}{l}\text { Schumann, } \\
\text { Pelttari, Doma, } \\
\text { Karavirta y } \\
\text { Häkkinen, } 2016\end{array}$ & 27 & $\begin{array}{l}\text { Corredores aficionados. } \\
\text { Edad: } 33 \pm 7\end{array}$ & $\begin{array}{l}\text { Comprobar las } \\
\text { mejoras } \\
\text { neuromusculares que } \\
\text { genera un } \\
\text { entrenamiento de } \\
\text { fuerza }\end{array}$ & $\begin{array}{l}\text {-Grupo resistencia + fuerza }(E+S) \text { en la } \\
\text { misma sesión } \\
\text {-Grupo resistencia (E) }\end{array}$ & $\begin{array}{l}24 \text { semanas. E+S realizó entre } \\
\text { cuatro y seis sesiones semanales } \\
\text { de resistencia más dos de fuerza. } \\
\text { S realizó el mismo trabajo de } \\
\text { resistencia sin entrenamientos de } \\
\text { fuerza }\end{array}$ & $\begin{array}{l}\text { El entrenamiento de fuerza } \\
\text { realizado después del trabajo de } \\
\text { resistencia no incrementó los } \\
\text { niveles de fuerza, ni generó } \\
\text { hipertrofia }\end{array}$ \\
\hline $\begin{array}{l}\text { Myers, Schneider, } \\
\text { Schmale y Hazell, } \\
2015\end{array}$ & 34 & $\begin{array}{l}\text { Mujeres sedentarias. } \\
\text { Edad: } 20.9 \pm 3.2\end{array}$ & $\begin{array}{l}\text { Comparar los efectos } \\
\text { de un entrenamiento } \\
\text { en circuito con un } \\
\text { entrenamiento } \\
\text { concurrente de fuerza } \\
\text { y resistencia sobre la } \\
\text { capacidad aeróbica y } \\
\text { anaeróbica }\end{array}$ & $\begin{array}{l}\text {-Grupo entrenamiento en circuito } \\
\text { (CIRCUIT) } \\
\text {-Grupo entrenamiento concurrente } \\
\text { tradicional (COMBINED) }\end{array}$ & $\begin{array}{l}\text { Cinco semanas. Tres sesiones } \\
\text { semanales }\end{array}$ & $\begin{array}{l}\text { Solo CIRUCIT mejoró la } \\
\text { capacidad aeróbica. Ambos } \\
\text { grupos mejoraron la capacidad } \\
\text { anaeróbica y la fuerza en } \\
\text { pectoral e isquiotibiales. Solo } \\
\text { COMBINED mejoró la fuerza en } \\
\text { espalda y cuádriceps }\end{array}$ \\
\hline $\begin{array}{l}\text { Eklund, } \\
\text { Pulverenti, } \\
\text { Bankers, Avela, } \\
\text { Newton, } \\
\text { Schumann y } \\
\text { Häkkinen, } 2015\end{array}$ & 56 & $\begin{array}{l}\text { Hombres con buena } \\
\text { salud. Edad: } 18-40\end{array}$ & $\begin{array}{l}\text { Evaluar las } \\
\text { adaptaciones } \\
\text { neuromusculares } \\
\text { logradas por medio de } \\
\text { dos entrenamientos } \\
\text { concurrentes de } \\
\text { fuerza y Pesistencia } \\
\text { realizados en la } \\
\text { misma sesión, pero en } \\
\text { orden inverso y un } \\
\text { entrenamiento } \\
\text { concurrente realizado } \\
\text { en días diferentes } \\
\end{array}$ & $\begin{array}{l}\text {-Grupo entrenamiento concurrente de } \\
\text { fuerza y lesistencia en días diferentes } \\
\text { (DD) } \\
\text {-Grupo entrenamiento combinado en la } \\
\text { misma sesión: Resistencia antes que } \\
\text { fuerza (E+S) } \\
\text {-Grupo entrenamiento combinado en la } \\
\text { misma sesión: Fuerza antes que } \\
\text { resistencia (S+E) }\end{array}$ & $\begin{array}{l}24 \text { semanas. DD de cuatro a seis } \\
\text { sesiones semanales. E+S y S+E } \\
\text { de dos a tres sesiones semanales }\end{array}$ & $\begin{array}{l}\text { Los tres grupos incrementaron la } \\
\text { fuerza dinámica, la hipertrofia y } \\
\text { la producción de potencia } \\
\text { máxima en ciclo. Sin embargo, } \\
\text { se observó que las adaptaciones } \\
\text { neuromusculares, (actividad } \\
\text { eléctrica ejercida por los } \\
\text { músculos esqueléticos), se ven } \\
\text { comprometidas con el protocolo } \\
\text { E+S }\end{array}$ \\
\hline
\end{tabular}

\begin{tabular}{|c|c|c|c|c|c|c|}
\hline Estudio & $\mathrm{N}$ & Grupo & Objetivo & Diseño Experimental & Duración & Resultados \\
\hline $\begin{array}{l}\text { Azeem y Hashem, } \\
2019\end{array}$ & 60 & $\begin{array}{l}\text { Estudiantes } \\
\text { Universitarios de } 18 \text { a } \\
22 \text { años }\end{array}$ & $\begin{array}{l}\text { Analizar el efecto de un programa } \\
\text { de entrenamiento de fuerza sobre } \\
\text { variables físicas y fisiologicas }\end{array}$ & $\begin{array}{l}\text {-Grupo experimental (A) } \\
\text {-Grupo control (B) }\end{array}$ & $\begin{array}{l}12 \text { semanas. Do } \\
\text { sesiones } \\
\text { semanales }\end{array}$ & $\begin{array}{l}\text { SEl grupo experimental mejoró la composición } \\
\text { corporal, la fuerza explosiva, la fuerza máxima, la } \\
\text { fuerza resistencia y la flexibilidad. También mejoró } \\
\text { la frecuencia cardiaca en reposo y el tiempo de } \\
\text { retención de la respiración }\end{array}$ \\
\hline $\begin{array}{l}\text { Morton, Whitehead, } \\
\text { Brinkert y Caine, } \\
2011\end{array}$ & 25 & Adultos no entrenados & $\begin{array}{l}\text { Comprobar el efecto del } \\
\text { entrenamiento de fuerza con rango } \\
\text { completo de movimiento y del } \\
\text { entrenamiento de flexibilidad } \\
\text { estático sobre los niveles de } \\
\text { flexibilidad y fuerza }\end{array}$ & $\begin{array}{l}\text {-Grupo de } \\
\text { o entrenamiento de fuerza de rango } \\
\text { completo de movimiento (RT) } \\
\text {-Grupo de de de } \\
\text { entrenamiento de de } \\
\text { flexibilidad estática (SS) }\end{array}$ & Cinco semanas & $\begin{array}{l}\text { Los entrenamientos de fuerza con rango completo de } \\
\text { movimiento, al igual que los estiramientos de } \\
\text { estáticos, pueden mejorar la flexibilidad }\end{array}$ \\
\hline Santos et al., 2010 & 24 & $\begin{array}{l}\text { Mujeres jóvenes } \\
\text { sedentarias. Edad: } \\
\text { Grupo AA: } 26.8 \pm 1.6 . \\
\text { Grupo AST: } 24 \pm 2.3 \\
\text { Grupo CG: } 25.4 \pm 2.4 \\
\end{array}$ & $\begin{array}{l}\text { Comprobar si el entrenamiento de } \\
\text { fuerza de intensidad moderada } \\
\text { mejora la flexibilidad en mujeres } \\
\text { jóvenes sedentarias }\end{array}$ & $\begin{array}{l}\text {-Grupo entrenamiento de fuerza: } \\
\text { Agonista/antagonista (AA) } \\
\text {-Grupo entrenamiento de fuerza: Tren } \\
\text { superior/ tren inferior (AST) } \\
\text {-Grupo control (CG) }\end{array}$ & $\begin{array}{l}\text { Ocho semanas. } \\
\text { Tres sesiones } \\
\text { semanales }\end{array}$ & $\begin{array}{l}\text { AA y AST aumentaron la fuerza y la flexibilidad } \\
\text { significativamente: (El incremento fue superior en } \\
\text { AST). Por tanto, el entrenamiento de fuerza mejoró } \\
\text { la flexibilidad en mujeres sedentarias jóvenes }\end{array}$ \\
\hline $\begin{array}{l}\text { Nóbrega, } \\
\text { Paula y } \\
\text { Carvalho, } 2005\end{array}$ & 43 & $\begin{array}{l}\text { Adultos con buena } \\
\text { salud. } \\
\text { Edad: } 21 \pm 4\end{array}$ & $\begin{array}{l}\text { Comprobar si los incrementos de } \\
\text { fuerza y flexibilidad se logran a } \\
\text { través de entrenamientos } \\
\text { especificos }\end{array}$ & $\begin{array}{l}\text {-Grupo entrenamiento de fuerza (a) } \\
\text {-Grupo entrenamiento de flexibilidad (b) } \\
\text {-Grupo de } \\
\text { entrenamiento de fuerza y flexibilidad (c) } \\
\text {-Grupo control (d) }\end{array}$ & $\begin{array}{l}12 \\
\text { semanas. Dos } \\
\text { sesiones } \\
\text { semanales }\end{array}$ & $\begin{array}{l}\text { El grupo "a" mejoró la fuerza. El grupo "b" mejoró la } \\
\text { flexibilidad. El grupo "c" mejoró la fuerza y la } \\
\text { flexibilidad. Por tanto, la aplicación del principio de } \\
\text { especificidad resulta esencial a la hora de mejorar los } \\
\text { niveles de fuerza muscular y de flexibilidad }\end{array}$ \\
\hline
\end{tabular}

contra, Nóbrega et al. (2005) hallaron que tan solo es posible conseguir adaptaciones de fuerza y flexibilidad cuando se aplican entrenamientos específicos dirigidos a la mejora de cada capacidad. Por lo tanto, se puede observar que la fuerza no solo no deteriora la flexibilidad, sino que incluso puede mejorarla. Santos et al. (2010) apuntan a que el entrenamiento de fuerza puede tener un impacto positivo en la flexibilidad cuanto la intensidad de trabajo es elevada. En este punto, cabría preguntarse entonces cuáles son las razones por las que el entrenamiento de fuerza, contrariamente a la creencia habitual, puede mejorar la flexibilidad. Uno de los motivos puede ser que un entrenamiento de fuerza correctamente ejecutado, en el que se realicen ejercicios dinámicos, (tanto concéntricos como excéntricos) y particularmente cuando se utiliza un rango completo de movimiento, implica la ejecución de un trabajo de movilidad que puede generar ciertas mejoras, especialmente en sujetos sedentarios. Otra explicación sería que la realización de estiramientos activos implica la ejecución de movimientos y el mantenimiento de determinadas posturas, y esto implica necesariamente la aplicación de fuerza, en concreto de la musculatura agonista, para flexibilizar los músculos antagonistas.

Finalmente, también conviene recordar que cada articulación posee un patrón de movimiento específico. (Bragança de Viana, Bastos de Andrade, Salguero del Valle, \& González-
Boto, 2008). Así pues, el impacto positivo del entrenamiento de fuerza sobre la flexibilidad, puede variar en virtud del test de flexibilidad empleado o de la articulación que sea objeto de valoración.

\section{Cuando el objetivo del entrenamiento es la hipertrofia muscular, las rutinas dividas son más eficaces que las ruti- nas de cuerpo entero}

Un aspecto de gran relevancia en el entrenamiento de fuerza, y que también genera controversia, es la estructura de la sesión de entrenamiento. Existen básicamente tres tipos de rutinas, (Heredia, Costa, Chulvi, Donate \& Soro, 2007; Kraemer \& Ratamess, 2004):

1) Rutinas globales o de cuerpo entero: Implican la realización de ejercicios que hacen hincapié en cada uno de los principales grupos musculares del cuerpo. Habitualmente se

incluye un ejercicio, o como máximo dos, para cada grupo muscular principal.

2) Rutinas dividas por hemisferios: En una sesión se realizan ejercicios destinados a fortalecer el tren superior del cuerpo, y en la siguiente, el tren inferior.

3) Rutinas divididas por grupos musculares: Implican la realización de ejercicios destinados al fortalecimiento de un reducido número grupos musculares en cada sesión.

Por tanto, las principales diferencias entre los tres tipos 


\begin{tabular}{|c|c|c|c|c|c|c|}
\hline Estudio & $\mathrm{N}$ & Grupo & Objetivo & Diseño Experimental & Duración & Resultados \\
\hline Zaroni et al., 2019 & 18 & $\begin{array}{l}\text { Adultos sanos y entrenados. } \\
\text { Edad: } 26.4 \pm 4.6\end{array}$ & $\begin{array}{l}\text { Comparar una rutina divida por } \\
\text { grupos musculares, con una rutina } \\
\text { de cuerpo entero }\end{array}$ & $\begin{array}{l}\text { Grupo entrenamiento cuerpo entero } \\
\text { (TOTAL) Grupo entrenamiento rutina } \\
\text { dividida por grupos musculares } \\
\text { (SPLIT) }\end{array}$ & $\begin{array}{l}\text { Ocho semanas. } \\
\text { Cinco sesiones semanales }\end{array}$ & $\begin{array}{l}\text { Ambos grupos obtuvieron } \\
\text { incrementos similares en sus niveles } \\
\text { de fuerza máxima. El grado de } \\
\text { hipertrofia alcanzado por el grupo } \\
\text { TOTAL fue superior al del grupo } \\
\text { SPLIT }\end{array}$ \\
\hline $\begin{array}{l}\text { Schoenfeld, } \\
\text { Ratamess, Peterson, } \\
\text { Contreras, } \\
\text { Tiryaki- Sonmez, } \\
2015\end{array}$ & 20 & $\begin{array}{l}\text { Estudiantes Universitarios } \\
\text { de sexo masculino y } \\
\text { entrenados. Edad: } 23.54 \pm 2.9\end{array}$ & $\begin{array}{l}\text { Valorar las } \\
\text { adaptaciones de fuerza logradas por } \\
\text { dos grupos de entrenamiento: Uno a } \\
\text { través de una rutina divida, y otro a } \\
\text { través de un entrenamiento de } \\
\text { cuerpo entero }\end{array}$ & $\begin{array}{l}\text {-Grupo rutina dividida (SPLIT): Cada } \\
\text { grupo muscular se estimuló una vez por } \\
\text { semana } \\
\text {-Grupo cuerpo entero (TOTAL): Cada } \\
\text { grupo muscular se estimuló } 3 \text { veces por } \\
\text { semana }\end{array}$ & $\begin{array}{l}\text { Ocho semanas. Tres sesiones } \\
\text { rsemanales } \\
\text { r }\end{array}$ & $\begin{array}{l}\text { El incremento de fuerza máxima } \\
\text { fue similar en ambos grupos. En } \\
\text { cambio, el grupo TOTAL obtuvo } \\
\text { mayores niveles de hipertrofia }\end{array}$ \\
\hline $\begin{array}{l}\text { Calder, Chilibeck, } \\
\text { Webber y Sale, } 1994\end{array}$ & 30 & $\begin{array}{l}\text { Mujeres jóvenes. Edad: } \\
\text { Grupo cuerpo entero: } \\
21.2 \pm 0.9 \text {. } \\
\text { Grupo rutina dividida: } \\
20.7 \pm 1.3 \text {. } \\
\text { Grupo control: } 20.7 \pm 0.6 \text {. }\end{array}$ & $\begin{array}{l}\text { Comprobar los niveles de fuerza } \\
\text { máxima e hipertrofia alcanzados a } \\
\text { través de dos entrenamientos de } \\
\text { fuerza diferentes: Uno de cuerpo } \\
\text { completo, y otro por medio de una } \\
\text { rutina dividida }\end{array}$ & $\begin{array}{l}\text {-Grupo entrenamiento de fuerza cuerpo } \\
\text { entero }(\mathrm{W}) \\
\text {-Grupo entrenamiento de fuerza rutina } \\
\text { dividida (S) } \\
\text {-Grupo control }\end{array}$ & $\begin{array}{l}\text { Dos períodos de } \\
10 \text { semanas separados por un } \\
\text { receso de dos semanas. } \\
\text { Numero de sesiones semanales: } \\
\text {-Grupo W: Dos } \\
\text {-Grupo S: Cuatro (dos días tren } \\
\text { superior y dos tren inferior) }\end{array}$ & $\begin{array}{l}\text { Ambos grupos experimentales } \\
\text { obtuvieron mejoras similares en sus } \\
\text { niveles de fuerza muscular, } \\
\text { hipertrofia muscular y } \\
\text { modificación de la composición } \\
\text { corporal }\end{array}$ \\
\hline
\end{tabular}

de sesión radican en:

a) El número de ejercicios por grupo muscular que se realizan en cada sesión.

b) En el número de sesiones semanales en las que se estimula un grupo muscular

concreto.

c) El tiempo de recuperación entre entrenamientos para cada grupo muscular.

Determinados autores argumentan que las rutinas divididas son más eficaces en el incremento del tejido muscular, debido a que permiten una mayor recuperación entre sesiones, y esto a su vez posibilita el uso de cargas de entrenamiento más elevadas, de modo que la tensión muscular es mayor. Sostienen además que las rutinas divididas pueden servir para aumentar el estrés metabólico muscular, al pro- longar el estímulo de entrenamiento dentro de un grupo muscular determinado, lo que puede potenciar las secreciones hormonales anabólicas agudas, la inflamación celular y la isquemia muscular. Otro de los argumentos empleados en favor de las rutinas divididas es que generan menor fatiga. (Calder, Chilibeck, Webber, \& Sale, 1994).

Por contra, los autores que se decantan por una rutina de cuerpo entero, mantienen que la respuesta hormonal al entrenamiento de fuerza está directamente vinculada a la cantidad de musculatura empleada en cada sesión de entrenamiento (Kraemer \& Ratamess, 2005). En la tabla 4, se muestran los resultados de tres estudios en los que se ha analizado que tipo de estructura de entrenamiento es más eficaz.

En los tres estudios se verificó que tanto las rutinas divididas como las de cuerpo completo son eficaces a la hora de

\begin{tabular}{|c|c|c|c|c|c|c|}
\hline Estudio & $\mathrm{N}$ & Grupo & Objetivo & Diseño experimental & Duración & Resultados \\
\hline $\begin{array}{l}\text { Castro-Jiménez, Gálvez- } \\
\text { Pardo, Guzmán-Quintero y } \\
\text { Garcia-Muñoz, } 2019\end{array}$ & $y^{27}$ & $\begin{array}{l}\text { Mujeres no fumadoras que } \\
\text { no padecían enfermedades. } \\
\text { Edad media: } 63 \text { años }\end{array}$ & $\begin{array}{l}\text { Analizar los efectos de un } \\
\text { programa de fuerza máxima sobre } \\
\text { la fuerza explosiva }\end{array}$ & e & $\begin{array}{l}12 \text { semanas. Tres } \\
\text { sesiones semanales }\end{array}$ & $\begin{array}{l}\text { El grupo experimental mejoró su } \\
\text { scomposición corporal de forma } \\
\text { significativa, pero no su fuerza explosiva }\end{array}$ \\
\hline $\begin{array}{l}\text { Knox, Sculthorpe y Grace, } \\
2019\end{array}$ & & $\begin{array}{l}\text { Hombres caucásicos y } \\
\text { surasiáticos. Edad: } \\
\text { Caucásicos: } 25.5 \pm 4.8 \text {; } \\
\text { Surasiáticos: } 25.4 \pm 7.0\end{array}$ & $\begin{array}{l}\text { Verificar el efecto de un } \\
\text { entrenamiento de fuerza sobre } \\
\text { biomarcadores cardiometabólicos }\end{array}$ & $\begin{array}{l}\text {-Grupo Caucásicos } \\
\text {-Grupo surasiáticos }\end{array}$ & $\begin{array}{l}\text { Seis semanas. Tres } \\
\text { sesiones semanales } \\
\end{array}$ & $\begin{array}{l}\text { Ambos grupos mejoraron la glucosa en } \\
\text { sayunas, los niveles de colesterol y el factor } \\
\text { de crecimiento endotelial vascular. La } \\
\text { proteína C reactiva se incrementó solo en el } \\
\text { grupo de surasiáticos }\end{array}$ \\
\hline Dinyer et al., 2019 & 33 & $\begin{array}{l}\text { Mujeres no entrenadas. } \\
\text { Edad: } 21.2 \pm 2.2\end{array}$ & $\begin{array}{l}\text { Analizar el efecto de dos } \\
\text { entrenamientos de fuerza de } \\
\text { diferente intensidad sobre la } \\
\text { fuerza y la composición corporal }\end{array}$ & $\begin{array}{l}\text {-Grupo entrenamiento de fuerza de } \\
\text { baja intensidad } \\
\text {-Grupo entrenamiento de fuerza de } \\
\text { alta intensidad }\end{array}$ & $\begin{array}{l}12 \text { semanas. Dos } \\
\text { sesiones semanales }\end{array}$ & $\begin{array}{l}\text { Ambos grupos mejoraron su fuerza } \\
\text { smáxima, pero no consiguieron incrementar } \\
\text { su tejido magro ni reducir su porcentaje de } \\
\text { grasa corporal }\end{array}$ \\
\hline $\begin{array}{l}\text { Franco, Carneiro, Alves, } \\
\text { Júnior, De Sousa y Orsatti, } \\
2019\end{array}$ & 32 & Mujeres. Edad: $24.3 \pm 4.8$ & $\begin{array}{l}\text { Comparar el efecto de dos } \\
\text { entrenamientos de fuerza de } \\
\text { diferente intensidad }\end{array}$ & $\begin{array}{l}\text {-Grupo entrenamiento de fuerza de } \\
\text { baja intensidad (LL) } \\
\text {-Grupo de entrenamiento de fuerza de } \\
\text { alta intensidad (HL) }\end{array}$ & $\begin{array}{l}\text { Nueve semanas. } \\
\text { La primera fue de } \\
\text { e familiarización. } \\
\text { Dos sesiones } \\
\text { semanales }\end{array}$ & $\begin{array}{l}\text { Ambos grupos mejoraron su fuerza } \\
\text { máxima. LL incrementó en mayor medida } \\
\text { su tejido magro }\end{array}$ \\
\hline Santos et al., 2018 & 75 & $\begin{array}{l}\text { Hombres con al menos seis } \\
\text { meses de experiencia en } \\
\text { entrenamientos de fuerza. } \\
\text { Edad: } 24.3 \pm 2.8\end{array}$ & $\begin{array}{l}\text { Comparar las modificaciones } \\
\text { logradas en el porcentaje de grasa } \\
\text { corporal y en la fuerza máxima } \\
\text { con la aplicación de cuatro } \\
\text { programas de entrenamiento de } \\
\text { fuerza diferentes }\end{array}$ & $\begin{array}{l}\text {-Grupo de control (GCO) } \\
\text {-Grupo isotónico (SIG) } \\
\text {-Grupo método bi-set (GBS) } \\
\text {-Grupo entrenamiento en circuito } \\
\text { (GCT) } \\
\text {-Grupo entrenamiento convencional } \\
\text { (GCV). }\end{array}$ & $\begin{array}{l}12 \text { semanas. Tres } \\
\text { sesiones semanales }\end{array}$ & $\begin{array}{l}\text { Todos los grupos experimentales lograron } \\
\text { smejorar sus niveles de fuerza máxima y } \\
\text { reducir su porcentaje de grasa corporal. } \\
\text { GCV obtuvo mejoras significativamente } \\
\text { inferiores con respecto a los restantes } \\
\text { grupos experimentales }\end{array}$ \\
\hline Cholewa et al., 2018 & 20 & $\begin{array}{l}\text { Mujeres universitarias que } \\
\text { no habían realizado } \\
\text { entrenamientos de fuerza } \\
\text { estructurados en los últimos } \\
\text { seis meses. Edad: } 20.3+1.5\end{array}$ & $\begin{array}{l}\text { Comparar las mejoras en los } \\
\text { niveles de fuerza, composición } \\
\text { corporal e hipertrofia logradas a } \\
\text { través de dos entrenamientos de } \\
\text { fuerza diferentes }\end{array}$ & $\begin{array}{l}\text {-Grupo entrenamiento de fuerza con } \\
\text { volumen moderado (ML) } \\
\text {-Grupo de entrenamiento de fuerza } \\
\text { con volumen elevado (HL) }\end{array}$ & $\begin{array}{l}\text { Nueve semanas. } \\
\text { Tres sesiones } \\
\text { semanales }\end{array}$ & $\begin{array}{l}\text { Ambos entrenamientos generaron } \\
\text { hipertrofia muscular y mejoras en los } \\
\text { niveles de fuerza y en la composición } \\
\text { corporal }\end{array}$ \\
\hline $\begin{array}{l}\text { Fernandez-del-Valle, } \\
\text { Short, Chung, McComb, } \\
\text { Kloiber, Naclerio y } \\
\text { Larumbe-Zabala, } 2018\end{array}$ & 26 & $\begin{array}{l}\text { Adultos con buen estado de } \\
\text { salud. Edad: } 18-30\end{array}$ & $\begin{array}{l}\text { Analizar los efectos de un } \\
\text { entrenamiento } \\
\text { intenso de fuerza sobre la } \\
\text { composición corporal, los niveles } \\
\text { de fuerza y los niveles séricos de } \\
\text { irisina }\end{array}$ & $\begin{array}{l}\text {-Grupo entrenamiento intenso de } \\
\text { fuerza } \\
\text {-Grupo control }\end{array}$ & $\begin{array}{l}\text { Tres semanas. Tres } \\
\text { sesiones semanales }\end{array}$ & $\begin{array}{l}\text { El entrenamiento generó mejoras en los } \\
\text { sniveles de fuerza y en la composición } \\
\text { corporal. No hubo cambios en las } \\
\text { concentraciones de irisina }\end{array}$ \\
\hline $\begin{array}{l}\text { Reza-Mohammadi, } \\
\text { Sadegh-Khoshnam y } \\
\text { Khoshnam, } 2018\end{array}$ & 47 & $\begin{array}{l}\text { Hombres sedentarios con } \\
\text { buena salud. Edad: Grupo } \\
\text { fuerza: } 48.16 \pm 5.68 ; \text { Grupo } \\
\text { resistencia: } 49.50 \pm 7.63 ; \\
\text { Grupo concurrente: } \\
46.72 \pm 4.19 ; \text { Grupo control: } \\
46.50 \pm 4.37\end{array}$ & $\begin{array}{l}\text { Analizar los efectos de diferentes } \\
\text { tipos de ejercicio sobre la } \\
\text { composición corporal y los } \\
\text { factores de riesgo cardiovascular }\end{array}$ & $\begin{array}{l}\text {-Grupo fuerza } \\
\text {-Grupo resistencia } \\
\text {-Grupo concurrente } \\
\text {-Grupo control }\end{array}$ & $\begin{array}{l}12 \text { semanas. } \\
\text { Grupo fuerza: Dos } \\
\text { sesiones semanales } \\
\text { el primer mes, y } \\
\text { después tres. } \\
\text { Grupo resistencia } \\
\text { y grupo } \\
\text { concurrente: Tres } \\
\text { sesiones semanales }\end{array}$ & $\begin{array}{l}\text { Los tres grupos experimentales mejoraron } \\
\text { su composición corporal y generaron } \\
\text { stambién mejoras en las moléculas de } \\
\text { adhesión intercelular, en las moléculas de } \\
\text { adhesión vasculares y en la proteína C- } \\
\text { reactiva }\end{array}$ \\
\hline $\begin{array}{l}\text { Yang, Bay, Wang, Huang, } \\
\text { Teo y Goh, } 2018\end{array}$ & & $\begin{array}{l}\text { Hombres jóvenes que } \\
\text { practicaban actividad física. } \\
\text { Edad: Grupo entrenamiento } \\
\text { en días consecutivos: } 24 \pm 2 ; \\
\text { Grupo entrenamiento en dias } \\
\text { no consecutivos: } 25 \pm 2\end{array}$ & $\begin{array}{l}\text { Examinar los efectos de } \\
\text { dos entrenamientos de fuerza } \\
\text { diferentes sobre los niveles de } \\
\text { fuerza, la composición corporal y } \\
\text { s los glóbulos rojos }\end{array}$ & $\begin{array}{l}\text {-Grupo entrenamiento de fuerza tres } \\
\text { días consecutivos (C) } \\
\text {-Grupo entrenamiento de fuerza en } \\
\text { días no consecutivos (NC) }\end{array}$ & $\begin{array}{l}12 \text { semanas. Tres } \\
\text { sesiones semanales } \\
\text { tanto C como NC }\end{array}$ & $\begin{array}{l}\text { Ambos protocolos de entrenamiento } \\
\text { sgeneraron mejoras similares en los niveles } \\
\text { de fuerza y en la composición corporal, e } \\
\text { indujeron a modificaciones similares en los } \\
\text { glóbulos rojos }\end{array}$ \\
\hline
\end{tabular}


incrementar los niveles de fuerza, y que no existen diferencias significativas entre ambas. Asimismo, Zaroni et al. (2019) y Schoenfeld et al. (2015) comprobaron que las rutinas de cuerpo entero generaron mayores niveles de hipertrofia. En ambos estudios cabe interpretar que la síntesis de proteínas musculares se vio favorecida por una mayor frecuencia semanal de entrenamiento, aun a pesar de que el número de series dedicadas a cada grupo muscular dentro de cada sesión fue reducido. Estos resultados sugieren que la hipertrofia muscular se ve favorecida cuando la frecuencia semanal con la que estimula un grupo muscular concreto es elevada, y que el volumen de entrenamiento empleado en cada sesión ejerce una menor influencia.

En cualquier caso, dado que tan solo existen tres investigaciones que se han ocupado de esclarecer cuál de las dos estructuras de entrenamiento es más eficaz, los resultados de estos estudios han de ser confirmados en futuras investigaciones.

\section{EI entrenamiento de fuerza carece de utilidad en los programas de pérdida de peso o de cambio de composi- ción corporal}

La eficacia del entrenamiento de fuerza a la hora de reducir el peso corporal ha sido a menudo cuestionada. A las personas que pretenden reducir su peso o su porcentaje de grasa corporal se les ha recomendado tradicionalmente la práctica de ejercicio aeróbico. En la tabla 5 se exponen los resultados de nueve estudios en los que se ha analizado la contribución del entrenamiento de fuerza a la mejora de parámetros cineantropométricos o de condición física y salud.

En siete de los nueve estudios analizados, el entrenamiento de fuerza generó mejoras en la composición corporal. Tan solo en uno de ellos, (Dinyer et al., 2019) los participantes no consiguieron incrementar su tejido magro ni reducir su porcentaje de grasa.

En el estudio realizado por Knox et al. (2019) no se evaluó el porcentaje de grasa corporal, pero se verificó que el entrenamiento de fuerza tuvo un impacto positivo en los niveles de glucosa y colesterol en ayunas y en el factor de crecimiento endotelial vascular. La proteína $\mathrm{C}$ reactiva se incrementó además en unos de los dos grupos experimentales.

Por su parte, Reza-Mohammadi at al. 2018, además de observar mejoras en la proteína $\mathrm{C}$ reactiva, también constataron mejoras en las moléculas de adhesión intercelular y vasculares.

\section{Conclusiones}

A través de este estudio se ha podido determinar que en personas adultas sanas:

1. El entrenamiento conjunto de fuerza y resistencia es compatible. Y si bien es cierto que puede atenuar la consecución de ciertas adaptaciones, es posible minimizar las posibles interferencias que surjan entre ambas capacidades separando en al menos 24 horas las sesiones de fuerza de las de resistencia, y evitando un excesivo volumen de entrenamiento. En caso que el trabajo de fuerza y resistencia se realice dentro de la misma sesión, cuando la sesión de fuerza precede a la de resistencia, se favorecen las adaptaciones de fuerza. Y si se invierte el orden, se favorecen las adaptaciones de resistencia.

2. El entrenamiento de fuerza no solo no deteriora los niveles de flexibilidad, sino que podría incluso incrementarlos. Sin embargo, se necesitan más estudios que permitan corroborar esta última posibilidad.

3. Aunque existen pocos estudios en los que se haya analizado que tipo de estructura de entrenamiento es más eficaz (rutinas divididas versus rutinas de cuerpo entero), la literatura científica existente nos indica que ambas son igualmente eficaces para incrementar los niveles de fuerza máxima, y que las rutinas de cuerpo completo generan mayores niveles de hipertrofia. Pero al igual que en el caso anterior, es preciso que esta cuestión sea confirmada en futuras investigaciones.

4. El entrenamiento de fuerza permite lograr mejoras en la composición corporal, y podría además ejercer un impacto positivo en determinados biomarcadores cardiovasculares y metabólicos.

\section{Referencias}

Amaro-Gahete, F.J., De-la-O, A., Jurado-Fasoli, L., DoteMontero, M., Gutiérrez, A., Ruiz, J.R., \& Castillo, M.J. (2019). Changes in physical fitness after 12 weeks of structured concurrent exercise training, high intensity interval training, or whole-body electromyostimulation training in sedentary middle-aged adults: A randomized controlled trial. Front physiol, 10(450), 1-15. doi: 10.3389/fphys.2019.00451

Azeem, K., \& Hashem, M.H. (2019). The effect of resistance training on the selected physical and physiological variables of the male students. International Journal of Pharmaceutical Research \& Allied Sciences, 8(2), 198205

Balsalobre-Fernández, C. \& Tejero-González, C.M. (2015). Efecto del entrenamiento con cargas sobre la grasa corporal en personas obesas: revisión sistemática. Revista Internacional de Medicina y Ciencias de la Actividad Física y el Deporte, 15(58), 371-386.

Bragança de Viana, M.M., Bastos de Andrade, A., Salguero del Valle, A., \& González-

Boto, R. (2008). Flexibilidad: conceptos y generalidades. Lecturas: Educación Física y Deportes, (Buenos

Aires), 12(116), Recuperado de http:// www.efdeportes.com/efd116/flexibilidad-conceptos-ygeneralidades.htm

Calder,A.W., Chilibeck, P.D., Webber, C.E., \& Sale, D.G.(1994). Comparison of whole and split weight training routines in young women. Can J Appl Physiol, 19(2), 185-99. doi: 10.1139/h94-014

Campos, F., González-Víllora, S., González, D., \& Martins, F.M.L. (2018). Retos, 35, 224-228

Castro, L.E., Gálvez,A.Y., Guzmán, G.A., \& Garcia,A.I. (2019). Fuerza explosiva en adultas mayores, efectos del entrenamiento en fuerza máxima. Retos, 36, 64-68.

Cholewa, J.M., Rossi, F.E., MacDonald, C., Hewins, A., Gallo, S.,...Campbell, B.I. (2018). The effects of moderate- versus high-load resistance training on muscle growth, body composition, and performance in collegiate women. $J$ 
Strength Cond Res, 32(6), 1511-1524. doi: 10.1519/ JSC.0000000000002048

Del Río, J.E., Flores, P.J., Bautista, J., Barajas, L.T., Valencia, R.T., Gómez, E. (2015). Efectos de un programa de flexibilidad en el desarrollo de la fuerza muscular en jugadoras de fútbol femenil. Educación Física y Ciencia, 17(2), 19

Dinyer, T.K., Byrd, M.T., Garver, M.J., Rickard, A.J., Miller, W.M.,...Bergstrom, H.C. Low-load vs. high-load resistance training to failure on one repetition maximum strength and body composition in untrained women. $J$ Strength Cond Res, 33(7), 1737-1744. doi: 10.1519/ JSC.0000000000003194

Eklund, D., Pulverenti, T., Bankers, S., Avela, J., Newton, R., Schumann, M., \& Häkkinen, K. (2016). Neuromuscular adaptations to different modes of combined strength and endurance training. Int J Sports Med, 36(2),120-9. doi: 10.1055/s-0034-1385883

Fernandez-del-Valle, M, Short, M.J., Chung, E., McComb, J., Kloiber, S., Naclerio, F., \& Larumbe-Zabala, E. (2018). Effects of high-intensity resistance training on circulating levels of irisin in healthy adults: A randomized controlled trial. Asian J Sports Med, 9(2), e13025. doi: 10.5812/ asjsm. 13025

Franco, C.M.C., Carneiro, M.A.D.S., Alves, L.T.H., Júnior, G.N.O., de Sousa, J.F.R., \& Orsatti, F.L. Lower-load is more effective than higher-load resistance training in increasing muscle mass in young women. $J$ Strength Cond Res, 33(Suppl 1), 152-158. doi: 10.1519/JSC.0000000000002970

Fry, A.C., \& Newton, R.U. (2006). Breve historia del entrenamiento de fuerza. Principios y conceptos básicos. En W.J. Kraemer, \& K. Häkkinen(Eds.), Entrenamiento de la fuerza (p. 18). Barcelona: Hispano Europea.

Fyfe, J.J., Bartlett, J.D., Hanson, E.D., Stepto, N.K., Bishop D.J. (2016). Endurance training intensity does not mediate interference to maximal lower-body strength gain during short-term concurrent training. Front Physiol, 7(487), 116 doi: 10.3389/fphys.2016.00487

Gäbler, M., Prieske, O., Hortobágyi, T., \& Granacher, U. (2018). The Effects of concurrent strength and endurance training on physical fitness and athletic performance in youth: A systematic review and meta-analysis. Front Physiol, 9(1057).

García-Orea, G.P., Heredia, J.R., Aguilera, J.A., Da Siva, M.E., \& Del Rosso, S. (2016). Entrenamiento concurrente de fuerza y resistencia: Una revisión narrativa. Journal of Physical Exercise and Health Science for Trainers, 1,1.

González-Badillo, J.J. \& Rivas, J. (2002). La fuerza y la resistencia. En J.J. González-Badillo \& J. Rivas (Eds.), Bases de la programación del entrenamiento de fuerza. (pp. 225-248). Barcelona: Inde.

Heredia, J.R., Costa, M.R., Chulvi, I., Donate, F.I, \& Soro, J. (2007). Determinación de la carga de entrenamiento para la mejora de la fuerza orientada a la salud. PubliCE. Recuperado de: https://g-se.com/determinacion-de-la-carga-de-entrenamiento-para-la-mejora-de-la-fuerza-orientada-a-la-salud-fitness-muscular-794-sa-K57cfb27188271

Karatrantou, K., Gerodimos, V., Häkkinen, K., \& Zafeiridis A. (2017). Health-promoting effects of serial vs. integrated combined strength and aerobic training. Int J Sports Med,
38(1), 55-64. doi: 10.1055/s-0042-116495

Knox, A., Sculthorpe, N., \& Grace, F. (2019). Caucasian and south Asian men show equivalent improvements in surrogate biomarkers of cardiovascular and metabolic health following 6-weeks of supervised resistance training. F1000Res, 7, 1334. doi: 10.12688/ f1000research.15376.2

Kraemer, W.J., \& Fleck, S.J. (2005). Strength Training and your child. En W.J. Kraemer, \& S.J. Fleck(Eds.), Strength training for young athletes. Second Edition. (pp. 5-6). Leeds: Human Kinetics.

Kraemer, W.J. \& Ratamess, N.A. (2004). Fundamentals of resistance training: progression and exercise prescription. Med Sci Sports Exerc, 36(4), 674-88. doi:10.1249/ 01.MSS.0000121945.36635.61

Kraemer, W.J. \& Ratamess, N.A. (2005). Hormonal responses and adaptations to resistance exercise and training. Sports Med, 35(4), 339-61

Küüsmaa-Schildt, M., Eklund, D., Avela, J., Rytkönen, T., Newton, R., Izquierdo, M., \& Häkkinen, K. (2017). Neuromuscular adaptations to combined strength and endurance training: Order and time-of-day. Int J Sports Med, 38(9),707-716. doi: 10.1055/s-0043-101376.

Küüsmaa-Schildt, M., Schumann, M., Sedliak, M., Kraemer, W.J., Newton, R., .. Nyman, K. (2016). Effects of morning versus evening combined strength and endurance training on physical performance, muscle hypertrophy, and serum hormone concentrations. Appl Physiol Nutr Metab, 41(12), 1285-1294

Laird, R.H., Elmer, D.J., Barberio, M.D., Salom, L.P., Lee, K.A., \& Pascoe, D.D. (2016). Evaluation of performance improvements after either resistance training or sprint interval-based concurrent training. J Strength Cond Res, 30(11), 3057-3065. doi: 10.1519/JSC.0000000000001412

Leveritt, M., Abernethy, P.J., Barry, B., \& Logan, P.A. (2003). Concurrent strength and endurance training: The influence of dependent variable selection. $J$ Strength Cond Res, 17(3),503-8. doi: 10.1519/15334287(2003)017<0503:csaett $>2.0 . c 0 ; 2$

-López-Miñarro, P.A. (2002). Conceptualización de mito, falsa creencia y práctica errónea. En P.A. López-Miñarro (Eds.), Mitos y falsas creencias en la práctica deportiva (pp. 15-24). Barcelona: Inde.

Mohammadi, H.R., Khoshnam, M.S., Khoshnam, E. Effects of different modes of exercise training on body composition and risk factors for cardiovascular disease in middle-aged men. Int J Prev Med, 9, 9. doi: 10.4103/ ijpvm.IJPVM_209_16

Morton, S.K., Whitehead, J.R., Brinkert, R.H., \& Caine, D.J. (2011). Resistance training vs. static stretching: Effects on flexibility and strength. J Strength Cond Res, 25(12), 3391-8. doi: 10.1519/JSC.0b013e31821624aa.

Myers, T.R., Schneider, M.G., Schmale, M.S., \& Hazell, T.J. (2015). Whole-body aerobic resistance training circuit improves aerobic fitness and muscle strength in sedentary young females. J Strength Cond Res, 29(6), 1592-600. doi: 10.1519/JSC.0000000000000790

Nóbrega, A.C., Paula, K.C., \& Carvalho, A.C. (2005). Interaction between resistance training and flexibility training in healthy young adults. J Strength Cond 
Res,19(4), 842-6. doi: 10.1519/R-15934.1

Peterson, M.D., Rhea, M.R., \& Alvar, B.A. (2004). Maximizing strength development in athletes: A meta-analysis to determine the dose-response relationship. Journal of Strength and Conditioning Research, 18(2), 377-82.

Pronk, P., Bender, E.G., \& Katz, A.S. (2016). Health, function, and performance benefits of workplace strength training programs. ACSM's Health \& Fitness Journal, 20(5), 6971. doi: 10.1249/FIT.0000000000000235

Robineau, J., Babault, N., Piscione, J., Lacome, M., \& Bigard, A.X. (2016). Specific

training effects of concurrent aerobic and strength exercises depend on recovery duration. J Strength Cond Res, 30(3), 672-83. doi: 10.1519/JSC.0000000000000798.

Sabag, A., Najafi, A., Michael, S., Esgin, T., Halaki, M., \& Hackett, D. (2018). The compatibility of concurrent high intensity interval training and resistance training for muscular strength and hypertrophy: A systematic review and meta-analysis. J Sports Sci, 36(21), 2472-2483.

Santos, E., Rhea, M.R., Simão, R., Dias, I., De Salles, B.F., Novaes, J.,... Bunker, D.J. (2010). Influence of moderately intense strength training on flexibility in sedentary young women. J Strength Cond Res, 24(11), 3144-9. doi: 10.1519/JSC.0b013e3181e38027.

Santos, L.C., Cherem, E.H., Azeredo, F.P., Neves, E.B., Oliveira, D.R.,....Novaes, J.S. (2018). Effects of different strength training programs in young males maximal strength and anthropometrics. Motricidade, 14(S1), 301-309

Schoenfeld, B.J., Ogborn, D., \& Krieger, J.W. (2016). Effects of resistance training frequency on measures of muscle hypertrophy: a systematic review and meta-analysis. Sports Med, 46(11), 1689-1697.

Schoenfeld, B.J., Ratamess, N.A., Peterson, M.D., Contreras, B., \& Tiryaki-Sonmez,

G. (2015). Influence of resistance training frequency on muscular adaptations in well-trained men. $J$ Strength

Cond Res, 29(7), 1821-9. doi:

$10.1519 /$ JSC.0000000000000970.

Schumann, M., Pelttari, P., Doma, K., Karavirta, L., \& Häkkinen, K. (2016). Neuromuscular adaptations to samesession combined endurance and strength training in recreational endurance runners. Int J Sports Med, 37(14),
1136-1143. doi: 10.1055/s-0042-112592

Sedano, S., Marín, P.J., Cuadrado, G., \& Redondo, J.C. (2013). Concurrent training in elite male runners: The influence of strength versus muscular endurance training on performance outcomes. J Strength Cond Res, 27(9), 243343. doi:10.1519/JSC.0b013e318280cc26.

Sousa, A.C., Marinho, D.A., Gil, M.H., Izquierdo, M., Rodríguez-Rosell, D., Neiva, H.P., Marques, M.C. (2018). Concurrent training followed by detraining: Does the resistance training intensity matter? J Strength Cond Res, 32(3), 632-642. doi: 10.1519/JSC.0000000000002237

Ten Hoor, G.A., Kok, G., Peters, G.Y., Frissen, T., Schols, A.M.W.J., \& Plasqui, G. The psychological effects of strength exercises in people who are overweight or obese: A systematic review. Sports med, 47(10), 2069-2081.

Thompson, W.R. (2018). Worldwide survey of fitness trends for 2019. ACSM's health \& fitness journal, 22(6), 10-17

Tomiya, S., Kikuchi, N., \& Nakazato, K. (2017). Moderate intensity cycling exercise after upper extremity resistance training interferes response to muscle hypertrophy but not strength gains. J Sports Sci Med, 16(3), 391-395

Torres, G., García-Martos, M., Villaverde, C., \& Garatachea, N. (2010). Papel del ejercicio físico en la prevención y tratamiento de la obesidad en adultos. Retos, (18)47-51.

Varela-Sanz,A., Tuimil, J.L.,Abreu, L., \& Boullosa, D.A. (2017). Does concurrent training intensity distribution matter? Journal of Strength and Conditioning Research, 31(1), 181-195. doi: 10.1519/JSC.0000000000001474

Weier, A.T, Pearce, A.J., \& Kidgell, D.J. (2012). Strength training reduces intracortical inhibition. Acta Physiol, 206(2),109-19. doi: 10.1111/j.1748-1716.2012.02454.X

Yang, Y., Bay, P.B., Wang, Y.R., Huang, J., Teo H.W.J., Goh, J. Effects of consecutive versus non-consecutive days of resistance training on strength, body composition, and red blood cells. Front Physiol, 18(9), 725. doi: 10.3389/ fphys.2018.00725

Zaroni, R.S., Brigatto, F.A., Schoenfeld, B.J., Braz, T.V., Benvenutti, J.C., Germano, M.D.,..Lopes, C.R. High resistance-training frequency enhances muscle thickness in resistance-trained men. J Strength Cond Res, 33 (Suppl 1), 140-151.doi: 10.1519/JSC.0000000000002643

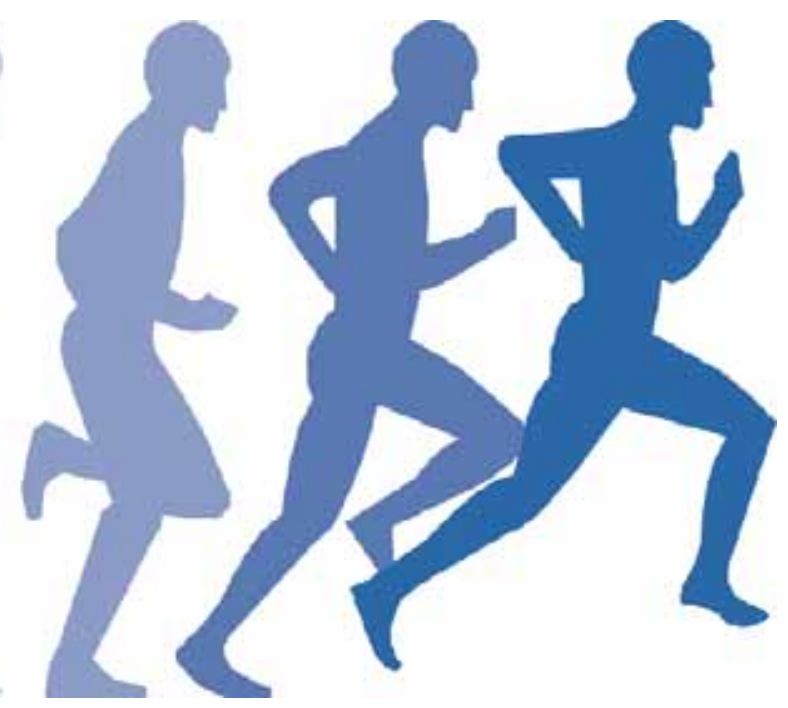

